\title{
NOUVELle
}

\section{Fin de l'orphelinat pour les récepteurs nucléaires REVERB}

Fabienne Guillaumond, Michèle Teboul
Laboratoire de Biologie

et de Physiopathologie des Systèmes Intégrés, CNRS FRE 3094, Université de Nice-Sophia Antipolis,

28, avenue Valrose, 06108 Nice Cedex 2, France.

fguillau@unice.fr
$>$ Identifiés depuis une vingtaine d'années, les récepteurs nucléaires REVERB, REVERB $\alpha$ (NRIDl) et REVERB $\beta$ (NRID2), sont dépourvus du domaine de transactivation AF2 dépendant du ligand et ont été classés parmi les récepteurs orphelins c'est-à-dire sans ligand connu. Ce sont des répresseurs transcriptionnels constitutifs qui se fixent sur des éléments de réponse de type WAWNTRGGTCA, appelés RORE [ROR (retinoid acid related orphan receptor) response elements]. Initialement, ce motif a été décrit comme le site de liaison des récepteurs nucléaires orphelins ROR, qui, eux, sont des activateurs transcriptionnels. L'activité répressive des REVERB résulte de la fixation du corépresseur N-CoR sur leur domaine de liaison au ligand (LBD), ce qui conduit à l'activation de la protéine HDAC (histone désacétylase) qui désacétyle les histones et rend ainsi la chromatine transcriptionnellement inactive.

\section{REVERB, régulateurs du métabolisme lipidique et constituants}

des oscillateurs circadiens

Les gènes Reverb $\alpha$ et $\beta$ présentent un patron d'expression spatio-temporel. Ils sont exprimés dans la plupart des tissus et sont particulièrement abondants dans le foie, le muscle, le tissu adipeux et le cervelet. Leur expression est rythmique dans de nombreux tissus. Les REVERB ont été impliqués dans divers processus biologiques, leur expression est induite au cours de la différenciation adipocytaire $[1,2]$ et diminuée au cours de la différenciation musculaire [3, 4]. Des expériences de génétique ont de plus montré que les REVERB ont un rôle important dans la régulation du métabolisme lipidique: des souris déficientes en REVERB $\alpha$ présentent une dyslipidémie avec des VLDL (very low density lipoproteins) élevées ainsi qu'une augmentation de l'expression de l'apolipoprotéine CIII [5]. Par ailleurs des cellules exprimant un dominant négatif de REVERB $\beta$ présentent une diminution de l'expression des gènes impliqués dans le métabolisme lipidique [6]. Outre leur rôle dans la régulation du métabolisme lipidique, les REVERB sont des constituants de l'oscillateur moléculaire des mammifères et donc des régulateurs du système circadien. L'invalidation du gène Reverb $\alpha$ chez la souris induit une diminution drastique de l'amplitude du rythme d'expression de Bmall, un composant moléculaire essentiel au fonctionnement de l'oscillateur circadien [7]. Celui-ci est constitué de boucles d'autorégulation transcriptionnelle et post-traductionnelle négatives mettant en jeu des gènes spécifiques (dits gènes-horloges) et leurs protéines respectives (BMAL1, CLOCK, CRYPTOCHROME 1 et 2, PERIOD 1 et 2) [8]. La robustesse de ce système est assurée par les récepteurs nucléaires, REVERB et ROR qui, respectivement, répriment et activent rythmiquement la transcription de Bmall [9]. Ces oscillateurs moléculaires imposent une rythmicité circadienne à de nombreuses fonctions physiologiques et comportementales de l'organisme, comme par exemple, le métabolisme lipidique et glucidique, la sécrétion hormonale (corticostérone, leptine, insuline) et le cycle veille-sommeil.

ع75, l'orthologue chez la drosophile des REVERB, est lié à l'hème La production dans un système hétérologue de récepteurs nucléaires conduit souvent à une co-purification de ces derniers avec de petites molécules qui les stabilisent. Cette approche peut permettre l'identification de ligands. C'est ainsi que Reinking et al., en exprimant dans $\varepsilon$. coli le LBD de $\varepsilon 75$ [10], l'orthologue unique chez la drosophile des deux REVERB, ont obtenu une protéine purifiée de couleur rouge, suggérant une co-purification avec un chromophore. Les analyses ont montré qu'il s'agissait de la protoporphyrine IX couplée à un ion $\mathrm{Fe}^{2+}$, communément appelée hème. L'hème est présent dans de nombreuses hémoprotéines (hémoglobine, myoglobine, cytochromes...), c'est un pigment organique rouge qui fixe et transporte l'oxygène et les gaz diatomiques ( $\mathrm{NO}$ et $\mathrm{CO}$ ). L'hème n'est pas à proprement parler un ligand de $\varepsilon 75$, puisqu'il est nécessaire à la stabilité de la protéine qui n'existe pas sous forme d'aporécepteur. L'état d'oxydation de l'hème détermine si $₹ 75$ peut dimériser avec son partenaire DHR3 suggérant un rôle de senseur redox. De plus, la dimérisation $875-D H R 3$ est aussi régulée par la fixation de $\mathrm{NO}$ ou de $C 0$ à l'hème, suggérant que $\varepsilon 75$ pourrait être également un senseur de gaz diatomiques. 


\section{Les REVERB sont aussi}

\section{des récepteurs de l'hème}

En se basant sur les travaux sur $\varepsilon 75$, les équipes de M.A Lazar [11] et de F. Rastinejad [12] ont purifié les domaines LBD de REVERB $\alpha$ et $\beta$ produits chez $\varepsilon$. Coli, et ont mis en évidence, par spectrométrie de masse, une interaction de l'hème avec ces deux récepteurs. Contrairement à ce qui se passe chez la drosophile, l'hème semble se comporter comme un ligand des REVERB, puisqu'il lie de façon réversible les $L B D$. La fixation de l'hème est requise pour le recrutement du co-répresseur $\mathrm{N}$-CoR et une mutation qui empêche la fixation de l'hème empêche également l'interaction avec $\mathrm{N}$-CoR [11, 12]. Contrairement à ce qui se passe chez la drosophile, les gaz diatomiques ne modulent pas l'activité de l'hème [12]. La découverte de l'hème comme ligand des REVERB pose la question du rôle de l'hème dans la régulation du métabolisme lipidique et dans le système circadien. En modulant la concentration intracellulaire en hème, les auteurs ont pu démontrer les effets engendrés par l'hème sur l'expression des gènes cibles de REVERB $\alpha$. Une déplétion en hème, par le succinyl acétone qui prévient l'incorporation de fer dans l'hème, induit une diminution de l'interaction de REVERB $\alpha$ d'une part avec le complexe N-CoR-HDAC3 [1], 12] et d'autre part avec l'élément RORE présent dans le promoteur du gène Bmall [12]. Par conséquent, l'expression du gène Bmall est augmentée [11, 12] ainsi que celle d'un autre gène cible de REVERB $\alpha$, Elovl3 [12], impliqué dans la lipogenèse. Inversement, l'ajout d'hémine induit une interaction stable de REVERB $\alpha$ avec N-CoR-HDAC3 et une baisse de l'expression de Bmall [11, 12] ainsi que de deux gènes codant pour des enzymes clés de la gluconéogenèse, la glucose-6-phosphatase (G6Pase) et la phosphoénolpyruvate kinase (PEPCK) [11, 13]. En revanche, l'inhibition de la synthèse de l'enzyme limitante dans la biosynthèse de l'hème, l'acide $\delta$-aminolévulinique synthase 1 (ALASI), par des ARN interférants induit une augmentation de l'expression de G6Pase [11]. Par cette même technologie, l'équipe de M.A. Lazar a également montré que la diminution de l'expression de PEPCK induite par l'hème est dépendante de REVERB $\alpha$. De plus, la production de glucose est également diminuée et résulte en partie de la réduction de l'expression de G6Pase et de PEPCK [11].

\section{La synthèse de l'hème}

suit un rythme circadien

La synthèse de l'hème à partir du succinyl CoA et de glycine se fait en plusieurs étapes (voir synthèse $H$. de Verneuil, ce numéro). L'étape limitante est catalysée par l'enzyme ALAS (aminolevulinic acid synthase) qui existe sous deux isoformes (ALASl ubiquitaire et ALAS2 exprimée seulement dans les précurseurs des cellules sanguines) (Figure 1). L’hème néo-synthétisé va exercer un rétrocontrôle négatif sur Alasl. L'expression d'Alasl suit un rythme circadien [14] et est augmentée par le co-activateur des récepteurs PPAR, PGC-l $\alpha$ (peroxisome prolifera-

Figure 1. Modèle de régulation de l'activité des récepteurs nucléaires REVERB par l'hème. La première étape de la synthèse de l'hème est catalysée par l'acide $\delta$-aminolévulinique synthase (ALAS) permettant la transformation du succinyl $\mathrm{Co} A$ et de la glycine en acide $\delta$-aminolévulinique (ALA). Alas est exprimé selon un rythme circadien et est induit en situation de jeûne par PGC-l $\alpha$ (co-activateur des récepteurs PPAR). Après plusieurs étapes successives, ALA est transformée en protoporphyrine IX. L'incorporation d'un atome de fer à la protoporphyrine conduit à la formation de l'hème, cette étape peut être inhibée par le succinyl acétone. L'hème néo-synthétisée va d'une part exercer un rétrocontrôle négatif sur Alas et d'autre part interagir avec le domaine de liaison du ligand (LBD) des récepteurs nucléaires REVERB. Cette liaison de l'hème permet: (1) l'interaction des REVERB avec les éléments de réponse RORE (WAWNTRGGTCA) par l'intermédiaire de leur domaine de liaison à l'ADN (DBD); et (2) le recrutement du co-répresseur N-CoR. Ce dernier, par l'intermédiaire de HDAC3, induit la désacétylation des histones et inhibe ainsi la transcription des gènes cibles des REVERB impliqués dans le métabolisme énergétique, le système circadien et la différenciation cellulaire (adipocytaire et musculaire).

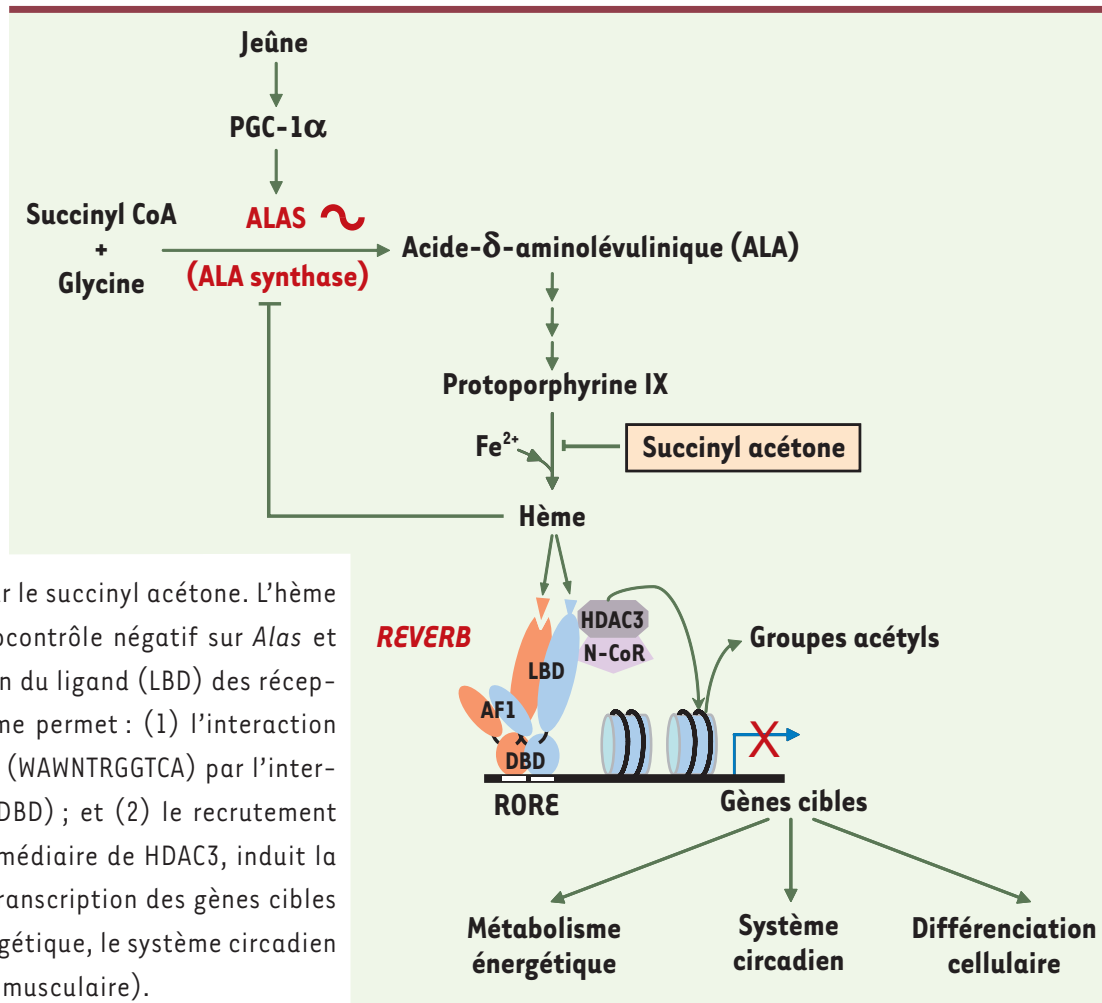


tor-activated receptor- $\gamma$ coactivator), lui-même régulé par le statut nutritionnel des mammifères (Figure 1).

\section{En conclusion}

Cela suggère que des altérations de l'expression d'Alasl peuvent conduire in fine à une modulation hème-dépendante de l'activité répressive des REVERB sur ses gènes cibles. Sachant que la synthèse de l'hème est rythmique, on peut dès lors envisager que l'hème, par son interaction avec les récepteurs REVERB situés à l'interface entre le système circadien, le métabolisme énergétique et la différenciation cellulaire, va exercer un contrôle rythmique sur les différents acteurs de ces processus physiologiques (Figure 1). $\diamond$ Heme as a ligand of REVERB $\alpha$ and $\beta$ nuclear receptors

\section{RÉFÉRENCES}

1. Chawla A, Lazar MA. Induction of Rev-ErbA alpha, an orphan receptor encoded on the opposite strand of the alpha-thyroid hormone receptor gene, during adipocyte differentiation. J Biol Chem 1993 ; $268: 16265-9$.

2. Wang J, Lazar MA. Bifunctional role of Rev-erb-alpha in adipocyte differentiation. Mol Cell Biol 2008 ; $28: 2213-20$.

3. Downes M, Carozzi AJ, Muscat GE. Constitutive expression of the orphan receptor, Rev-erbA alpha, inhibits muscle differentiation and abrogates the expression of the myoD gene family. Mol Endocrinol $1995 ; 9: 1666-78$.

4. Burke L, Downes M, Carozzi A, et al. Transcriptional repression by the orphan steroid receptor RVR/Rev-erb beta is dependent on the signature motif and helix 5 in the $\varepsilon$ region: functional evidence for a biological role of RVR in myogenesis. Nucleic Acids Res 1996 ; $24: 3481-9$

5. Raspé $\varepsilon$, Duez $H$, Mansén $A$, et al. Identification of Rev-erbalpha as a physiological repressor of apoC-III gene transcription. J Lipid Res $2002 ; 43: 2172-9$.

6. Ramakrishnan SN, Lau P, Burke LJ, Muscat GE. Reverbbeta regulates the expression of genes involved in lipid absorption in skeletal muscle cells: evidence for cross-talk between orphan nuclear receptors and myokines. J Biol Chem 2005 ; 280 : 8651-9.
7. Teboul M, Delaunay F. Le récepteur nucléaire orphelin Rev-erbalpha oscille entre répression et activation. Med Sci (Paris) 2003 ; $19: 411$-3.

8. Dardente $\mathrm{H}$. Redondance génétique et synchronisation cellulaire dans les horloges circadiennes. Med Sci (Paris) $2008 ; 24: 270-6$.

9. Guillaumond F, Dardente $\mathrm{H}$, Giguère $\mathrm{V}$, et al. Differential control of Bmall circadian transcription by REV-ERB and ROR nuclear receptors. J Biol Rhythms $2005 ; 20: 391-403$.

10. Reinking J, Lam MM, Pardee K, et al. The Drosophila nuclear receptor $\varepsilon 75$ contains heme and is gas responsive. Cell $2005 ; 122$ : 195-207.

11. Yin L, Wu N, Curtin JC, et al. Rev-erb-alpha, a heme sensor that coordinates metabolic and circadian pathways. Science 2007 ; 318 : $1786-9$.

12. Raghuram S, Stayrook KR, Huang P, et al. Identification of heme as the ligand for the orphan nuclear receptors REV-ERBalpha and REV-ERBbeta. Nat Struct Mol Biol 2007; 14 : 1207-13.

13. Cadoudal T, Fouque F, Benelli C, Forest C. Glycéronéogenèse et PEPCK-C : cibles pharmacologiques dans le diabète de type 2. Med Sci (Paris) $2008 ; 24: 407-14$.

14. Kaasik K, Lee CC. Reciprocal regulation of haem biosynthesis and the circadian clock in mammals. Nature $2004 ; 430$ : 467-71.

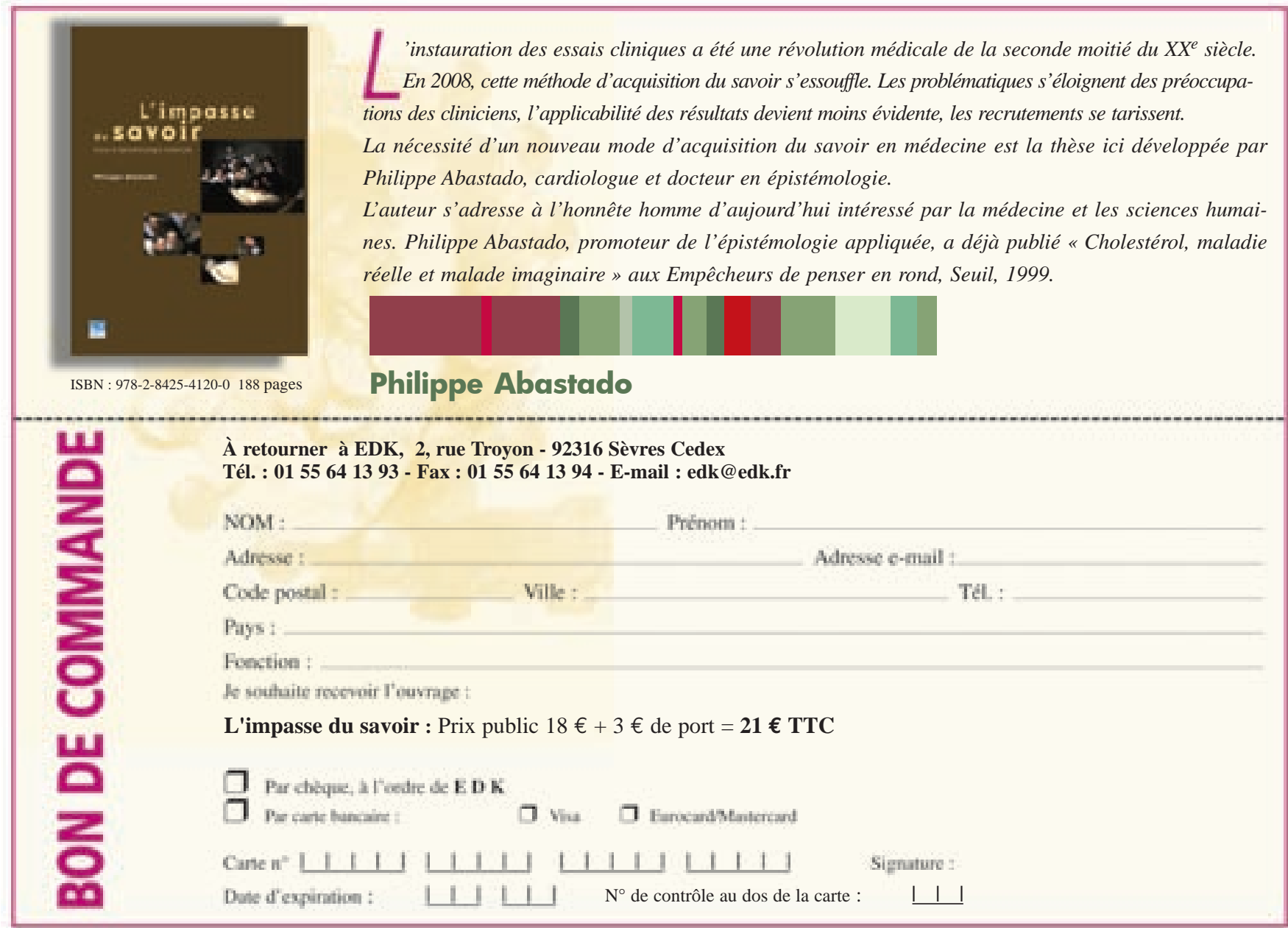

